\title{
Estimation of Fetal Brain Volume from MRI of Human Fetus
}

\author{
Gayathri SP ${ }^{1}$, Praveenkumar $S^{2}$, Somasundaram $\mathbf{K}^{* 3}$, Siva Shankar $\mathbf{R}^{4}$, Klaiselvi $\mathbf{T}^{5}$ and Magesh $\mathbf{S}^{\mathbf{6}}$ \\ ${ }^{1}$ Department of M Com, PSGR Krishnammal College for Women, India \\ ${ }^{2}$ Mirafra Inc., 3013 Fountainview Street, Houston, USA \\ ${ }^{4}$ Department of Computer Applications, Madanapal le Institute of Technology and Science, India \\ 3,5 Department of Computer Science and Applications, Gandhigram Rural Institute, India \\ ${ }^{6}$ IGS Global Soft Inc, Irving, USA
}

Received: 制: September 07, 2018; Published: 制 September 21, 2018

*Corresponding author: K Somasundaram, Department of Computer Science and Applications, Gandhigram Rural Institute, Gandhigram 624302, India

\section{Abstract}

Monitoring of fetal brain growth during the pregnancy period is essential to have a healthy fetus. Fetal brain volume is a biomarker to identify any deformity in the fetal brain. In this paper we propose a method to estimate the fetal brain volume by manually segmenting the MRI of human fetus.

Keywords: Fetal Brain; Fetal Brain Volume; Fetal MRI

Abbreviations: GW: Gestational Week; CNS: Central Nervous System; US: Ultrasound; MRI: Magnetic Resonance Imaging; TOP: Termination of Pregnancy; IUFD: In Utero Fetal Death: SSFSE: Single Shot Fast Spin Echo; FOV: Field of View; ST: Slice Thickness; ISG: Inter Slice Gap

\section{Introduction}

The human fetus undergoes rapid changes in every gestational week (GW). The fetus is to be monitored by an obstetrician for its healthy growth. An important organ that is to be monitored is the fetal brain. Fetal brain starts developing from the 3rd GW and continue to grow in its shape and structure [1]. Some of the abnormalities that may occur in the central nervous system (CNS) in the early stages are encephalocele, anencephaly, hydrocephalus and microcephaly [2]. To understand the pathology and structure of the fetus, non-invasive imaging techniques such as ultrasound (US) and magnetic resonance imaging (MRI) are used. MRI is more suitable to image soft tissues such as brain and also gives a high contrast image. Identification of brain abnormalities helps to make informed decisions like termination of pregnancy (TOP) or in utero fetal death (IUFD) [3]. Votino et al. [3] have estimated the brain volume using MRI and weight using autopsy of the fetal brain. Estimation of fetal brain at different GW helps to identify normal and abnormal brain growth [4,5]. Estimating the brain volume through MR imaging has become an accepted technique [6,7] In this paper, we propose a method to estimate the fetal brain volume from MRI of human fetus in the range of 20-38 GW by extracting the brain portion manually by an expert.

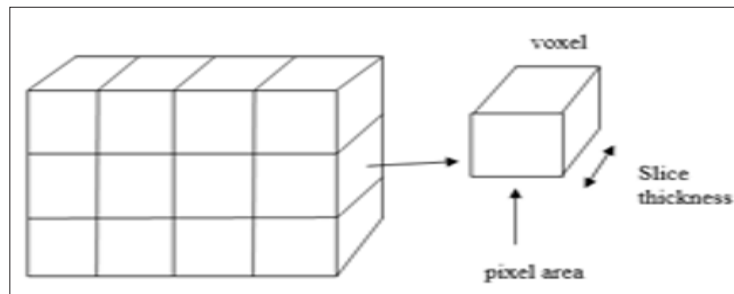

(a)

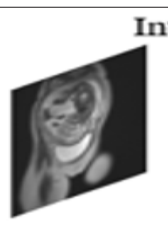

slice $\mathbf{n}-1$

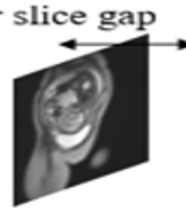

slice $n$

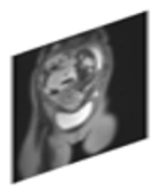

slice $\mathbf{n}+\mathbf{1}$

Figure 1: Slice terminology.

(a) Image matrix; (b) Stack of slices. 


\section{Materials Used}

We used twenty volumes of retrospective T2 - weighted singleshot fast spin echo (SSFSE) MRI collected from Sri Ramachandra Medical University and Hospital, Chennai, India. Ethical approval was granted by the Ethical Committee (ethics no. IEC-NI/14/ ОСТ/43/65) of the same University. The datasets were imaged between 20 and $38 \mathrm{GW}$. Brain portion in all the volumes were manually segmented by a medical expert specialized in the fetal brain anatomy and are used for estimating the brain volume. The details of the dataset are given in Table 1. The data sets pertain to different subjects. This set was arranged to cover range of 20-38

Table 1: Details of retrospective T2- $\mathrm{w}$ fetal image.

\begin{tabular}{|c|c|c|c|c|c|c|c|}
\hline \multirow{2}{*}{ No. } & \multirow{2}{*}{ GW } & \multirow{2}{*}{ Flip Angle } & \multirow{2}{*}{$\begin{array}{c}\text { Slice Thickness } \\
\text { (mm) }\end{array}$} & \multirow{2}{*}{ Slice gap $(\mathrm{mm})$} & TR & TE & FoV \\
\hline & & & & & (ms) & (ms) & $(\mathrm{mm})$ \\
\hline 1 & $20-21$ & 170 & 4 & 4 & 850 & 86 & $188 \times 280$ \\
\hline 2 & $20-21$ & 170 & 3.5 & 3.5 & 1350 & 89 & 280 \\
\hline 3 & 20 & 170 & 3.5 & 3.5 & 800 & 89 & 270 \\
\hline 4 & 29 & 90 & 4.5 & 5 & 718 & 92 & 350 \\
\hline 5 & 36 & 170 & 3.5 & 3.5 & 1790 & 89 & 270 \\
\hline 6 & 31 & 90 & 4.5 & 4.8 & 473 & 91 & 380 \\
\hline 7 & 29 & 170 & 4 & 4.2 & 1350 & 91 & 300 \\
\hline 8 & 27 & 90 & 5 & 5.2 & 642 & 93 & 350 \\
\hline 9 & 26 & 170 & 4.5 & 4.5 & 700 & 89 & 280 \\
\hline 10 & 23 & 170 & 4 & 4.2 & 1350 & 91 & 320 \\
\hline 11 & 29 & 170 & 4 & 4.2 & 1350 & 91 & 300 \\
\hline 12 & 33 & 90 & 4 & 4 & 1062 & 88 & 320 \\
\hline 13 & $20-21$ & 170 & 5 & 5.5 & 1350 & 91 & 320 \\
\hline 14 & $35-36$ & 170 & 4 & 4 & 1350 & 91 & 300 \\
\hline 15 & 38 & 90 & 4 & 4.5 & 676 & 88 & 400 \\
\hline 16 & $28-29$ & 170 & 4 & 4.2 & 1350 & 91 & 280 \\
\hline 17 & 31 & 90 & 3.5 & 3.7 & 709 & 90 & 420 \\
\hline 18 & $32-33$ & 160 & 3 & 3 & 900 & 89 & $279 \times 350$ \\
\hline 19 & $22-23$ & 160 & 3.5 & 3.9 & 1000 & 85 & $350 \times 279$ \\
\hline 20 & $35-36$ & 90 & 4 & 4.5 & 695 & 92 & 420 \\
\hline
\end{tabular}

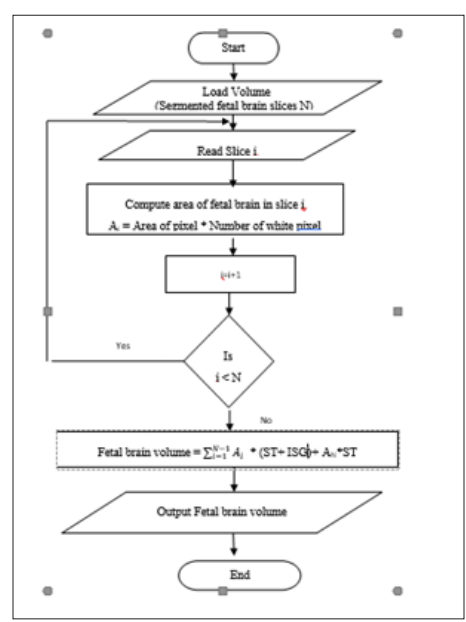

Figure 2: Flowchart of the proposed method. 


\section{Method}

The proposed method includes computing fetal brain area in each slice and estimating volume of fetal brain. The flowchart of the proposed method is shown in Figure 2.

\section{Computing Area of Fetal Brain in Each Slice}

For each segmented fetal brain from MRI slice, a binary mask is obtained. The pixels which have the intensity values greater than zero are assigned to one (white) and the rest of the pixels are assigned a zero value (black). The area is computed by counting the white pixels. In order to compute the area of the fetal brain in a slice, first the area of each pixel is computed in $\mathrm{cm}^{2}$. This is done by reading the pixel spacing tag from the DICOM header of the corresponding MRI slice. The pixel spacing tag represents the spacing between the centers of any two adjacent pixels [8]. Half the length of the pixel spacing represents half the length of a pixel and the other half represents half the length of the adjacent pixel. Assuming that all the pixels in the image have the same length in both $\mathrm{x}$ and $\mathrm{y}$ direction, the pixel spacing (in $\mathrm{mm}$ ) is equal to the pixel length. The area of any pixel is computed as:

$$
\text { Area of pixel }=\left(\frac{\text { pixelspacing }}{10}\right)^{2} \mathrm{~cm}^{2}
$$

Figure 3 shows that the pixel spacing is equal to the pixel length

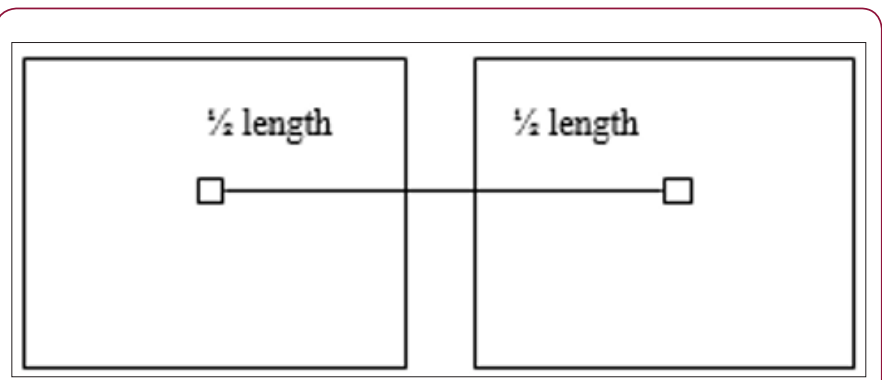

Figure 3: Pixel spacing is equal to pixel length.

The area of the brain in the $\mathrm{i}^{\text {th }}$ slice is computed as:

$$
\mathrm{Ai}=\text { Area of pixel } * \mathrm{~N}_{\mathrm{w}}
$$

where, $\mathrm{N}_{\mathrm{w}}$ is the total number of white pixel in the slice. This process is repeated for all the slices in that volume.

\section{Computing Fetal Brain Volume}

The brain volume is computed by multiplying the area and slice thickness (ST). While acquiring the images, a physical gap is left in between two slices and is measured by inter slice gap (ISG). It is assumed that the area of the brain in the ISG is the average of the two adjacent slices. The ST and ISG are obtained by reading the header of DICOM images. The fetal brain volume BV is computed as:

$$
B V=\sum_{i=1}^{N-1} A_{i} *(S T+I S G)+A_{N} * S T m L
$$

where, $\mathrm{N}$ is the total number of slices in the volume and $A_{i}$ represents the area of the fetal brain in the $\mathrm{i}^{\text {th }}$ slice, and $\mathrm{A}_{\mathrm{N}}$ is the $\mathrm{N}^{\text {th }}$ slice.

\section{Results and Discussion}

The computed values of BV for 12 different gestational weeks are given in Table 2 .

Table 2: Computed Fetal brain volume BV (mL).

\begin{tabular}{|c|c|c|}
\hline Vol Label & GW (weeks) & BV in $\mathbf{~ L ~}$ \\
\hline 1 & 20 & 66.84 \\
\hline 2 & 21 & 75.2 \\
\hline 3 & 22 & 81.3 \\
\hline 4 & 23 & 90.1 \\
\hline 5 & 26 & 112.1 \\
\hline 6 & 27 & 117.4 \\
\hline 7 & 29 & 132.87 \\
\hline 8 & 31 & 145.65 \\
\hline 9 & 32 & 158.27 \\
\hline 10 & 33 & 201.6 \\
\hline 11 & 36 & 320.2 \\
\hline 12 & 38 & 439.17 \\
\hline
\end{tabular}

\section{Conclusion}

We have developed a method to estimate the fetal brain volume from hand segmented brain portion from the MRI of human fetus. The fetal brain volumes at different gestational week were estimated. We found that the brain volume increases almost linearly till32 GW and thereafter at a higher rate. It is $67 \mathrm{~mL}$ at $20 \mathrm{GW}, 114 \mathrm{~mL}$ at $26 \mathrm{GW}$ and $438 \mathrm{~mL}$ at $38 \mathrm{GW}$.

\section{References}

1. Stiles J, Jernigan TL (2010) The Basics of Brain Development. Neuropsychol Rev 20(4): 327-348.

2. https://www.cdc.gov/ncbddd/birthdefects/encephalocele.html.

3. Votino C, Verhoye M, Segers V, Cannie M, Bessieres B, et al. (2012) Fetal organ weight estimation by postmortem high-field magnetic resonance imaging before 20 weeks' gestation. Ultrasound Obstet Gynecol 39(6): 673-678.

4. Glenn OA, Barkovich AJ (2006) Magnetic resonance imaging of the fetal brain and spine: an increasingly important tool in prenatal diagnosis, Part 1, American Journal of Neuroradiology 27(8): 1604-1611.

5. Prayer D, Kasprian G, Krampl E, Ulm B, Witzani L, et al. (2006) MRI of normal fetal brain development. European journal of radiology 57 (2): 199-216.

6. Gong QY, Roberts N, Garden AS, Whitehouse GH (1998) Fetal and fetal brain volume estimation in the third trimester of human pregnancy using gradient echo MR imaging. Magnetic Resonance Imaging 16(3): 235-240.

7. Gayathri SP (2016) Segmentation and volume estimation of fetal brain from T2-w magnetic resonance images (MRI) of human fetus, Ph.D. Thesis. The Gandhigram Rural Institute Tamil Nadu, India.

8. Le Bihan D, Mangin JF, Poupon C, Clark CA, Pappata S, et al. (2003) Diffusion tensor imaging: concepts and applications. Journal of magnetic resonance imaging 13(4): 534-546. 


\section{ISSN: 2574-1241}

DOI: $10.26717 / B J S T R .2018 .09 .001774$

K Somasundaram. Biomed J Sci \& Tech Res

(c) This work is licensed under Creative

Submission Link: https://biomedres.us/submit-manuscript.php

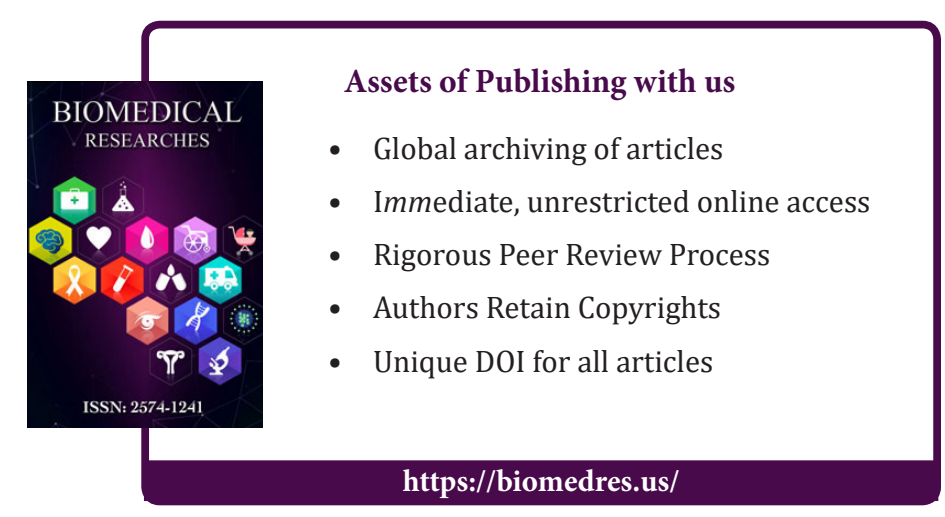

